\title{
Low-profile and wearable energy harvester based on plucked piezoelectric cantilevers
}

\author{
Michele Pozzi $^{* a}$, Heather J.A. Almond ${ }^{\mathrm{b}}$, Glenn J.T. Leighton ${ }^{\mathrm{b}}$, Roy J. Moriarty ${ }^{\mathrm{a}}$ \\ aSchool of Mechanical and Systems Engineering, Newcastle University, NE1 7RU, UK \\ ${ }^{\mathrm{b}}$ Cranfield University, Cranfield, MK43 0AL, UK
}

\begin{abstract}
The Pizzicato Energy Harvester (EH) introduced the technique of frequency up-conversion to piezoelectric EHs wearable on the lateral side of the knee-joint. The operation principle is to pluck the piezoelectric bimorphs with plectra so that they produce electrical energy during the ensuing mechanical vibrations. The device presented in this work is, in some ways, an evolution of the earlier Pizzicato: it is a significantly more compact and lighter device; the central hub holds 16 piezoelectric bimorphs shaped as trapezoids, which permits a sleek design and potentially increased energy output for the same bimorph area.

Plectra were formed by Photochemical Machining of a 100- $\mu$ m-thick steel sheet. To avoid the risk of short-circuiting, the plectra were electrically passivated by sputtering a $100 \mathrm{~nm}$ layer of $\mathrm{ZrO}_{2}$. Bench tests with the steel plectra showed a very large energy generation. Polyimide plectra were also manufactured with a cutting plotter from a $125 \mu \mathrm{m}$-thick film. Besides bench tests, a volunteer wore the device while walking on flat ground or climbing stairs, with a measured energy output of approximately $0.8 \mathrm{~mJ}$ per step.

Whereas most of the tests were performed by the traditional method of discharging the rectified output from the EH onto a resistive load, tests were performed also with a circuit offering a stabilised $3.3 \mathrm{~V}$ supply. The circuit produced a stable $0.1 \mathrm{~mA}$ supply during running gait with kapton plectra.
\end{abstract}

Keywords: energy harvesting, piezoelectric bimorph, piezoelectric cantilever, wearable energy, frequency upconversion, plucking, photochemical machining, personal energy harvester.

\section{INTRODUCTION}

Energy harvesting, also referred to as energy scavenging, is the process of converting small amounts of environmental energy into electrical energy with the goal of powering small and power efficient sensors ${ }^{1,2}$. Many sources of environmental energy have been investigated and exploited in recent years, such as vibrations ${ }^{3}$, thermal gradients ${ }^{4}$ and radio frequency signals ${ }^{5}$. When motion is present, such as in vibrations, the most common material used for energy transduction is a piezoelectric ceramic, such as PZT. The piezoelectric bimorph, with its good compliance, is advantageous in the process of matching the transducer to the mechanical impedance of the vibrations source. Nevertheless, short of employing very long and slender bimorphs with large tip masses, the natural frequencies of bimorphs is often higher than the available vibrations. This is particularly true for wearable energy harvesters (EHs) as human motions are characterised by very low frequencies in the order of hertz.

A solution to the frequency mismatch between source and transducer is represented by frequency up-conversion, introduced to energy harvesting a two decades ago $^{6}$ in the form of impact excitation and more recently as plucking of cantilevered beams ${ }^{7}$. These principles have been used for energy harvesting from human motion by several authors ${ }^{8,9}$. One of the present authors, in particular, has conceived and designed an EH based on plucking of piezoelectric bimorphs to be worn on the outside of the knee-joint ${ }^{10}$. This early design had the potential of producing around $2 \mathrm{~mJ}$ of electrical energy per step during normal walking. It was however intended as a proof of concept and therefore rather heavy and bulky.

This paper presents energy conversion results for a more compact EH which was also tested by a volunteer subject.

* Contact author: michele.pozzi@ncl.ac.uk

Smart Sensors, Actuators, and MEMS VII; and Cyber Physical Systems, edited by José L. Sánchez-Rojas,

Riccardo Brama, et. al., Proc. of SPIE Vol. 9517, 951706 - (c) 2015 SPIE

CCC code: $0277-786 \mathrm{X} / 15 / \$ 18 \cdot$ doi: $10.1117 / 12.2179574$

Proc. of SPIE Vol. 9517 951706-1 


\section{METHODOLOGY}

\section{Design}

As a step towards a higher Technology Readiness Level from the earlier Pizzicato $\mathrm{EH}^{10}$, the design of this prototype was guided by manufacturability. Whereas in the previous design the bimorphs were held in the hub by easily demountable mechanical fixings, permanent adhesive fixing was used in the present variant to achieve a higher density of bimorphs (increased from 4 to 16). Whereas the present hub was machined out of aluminium alloy, in industrial manufacture it would be cost effective to mould a polymeric hub with embedded bimorphs. Electrical contacts can then be embedded at the root as part of the moulding process. The bimorphs used have a trapezoidal (almost triangular shape), which has been shown to yield advantages for EH in terms of curvature homogenization ${ }^{11}$; since the strain is more uniform along the length, the piezoelectric material is used more efficiently. In the plucking excitation mode, the drawback associated with the position of the centroid discussed by Goldschmidtboeing and Woias does not apply (mechanical energy is introduced by the plectrum as a direct force near the tip). Furthermore, the shape is particularly advantageous in this application as it permits a sleeker overall design. The overall volume and mass of the energy harvester are $66 \mathrm{~cm}^{3}$ and $0.10 \mathrm{~kg}$ respectively, resulting in a significantly more compact and lighter device than the previous Pizzicato (0.24 kg and 230 $\left.\mathrm{cm}^{3}\right)$.

The other critical mechanical component is the set of plectra. For this, Photochemical Machining (PCM) was selected because of its capability to produce accurate parts from thin sheet material. Successive forming with custom made tooling (as would be needed here to lift off the plectra) is also common and reliable industrial manufacturing procedure. As alternative, plectra can be made of kapton or other suitable polymer sheet; in this case, cutting can be performed with a simple custom tool as part of an industrial process.

PCM is an accelerated but controlled corrosion process whereby a chemical medium (an etchant) is used to dissolve, mainly, metal substrates in a selective manner to create complex geometries ${ }^{12}$. "Selectivity" is achieved by using a photolithographic process to create patterned mask (resist) areas that are protected from subsequent etching. The process steps are detailed in Figure 1.

A phototool (a high resolution image of the required design consisting of transparent and opaque regions) is created using CAD. The metal substrate is cleaned thoroughly to ensure good adherence during the subsequent coating stage

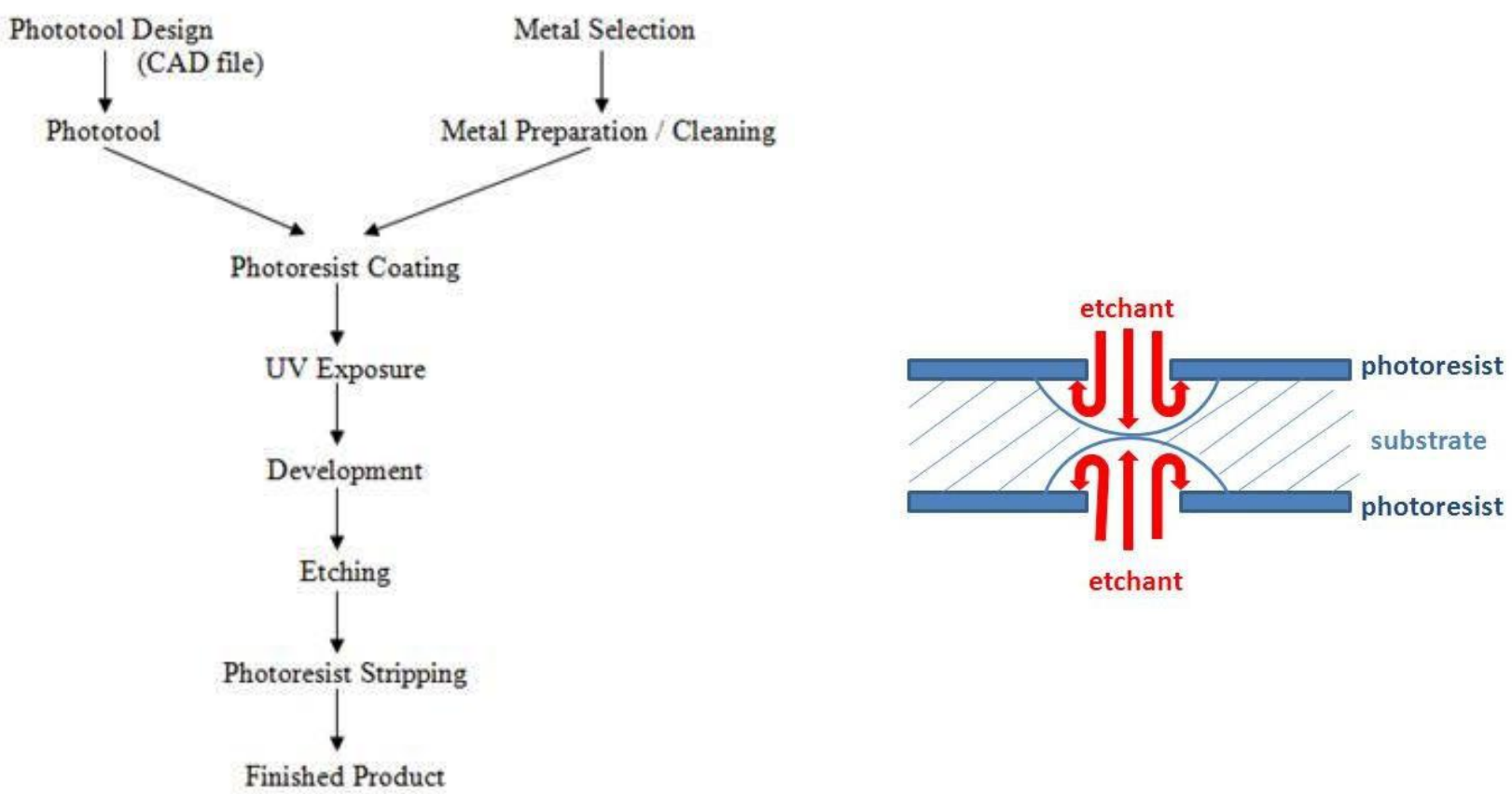

Figure 1: (left) Stages of the PCM process ${ }^{12}$. (right) Cross section through an etched profile in PCM showing the development of an etched aperture 

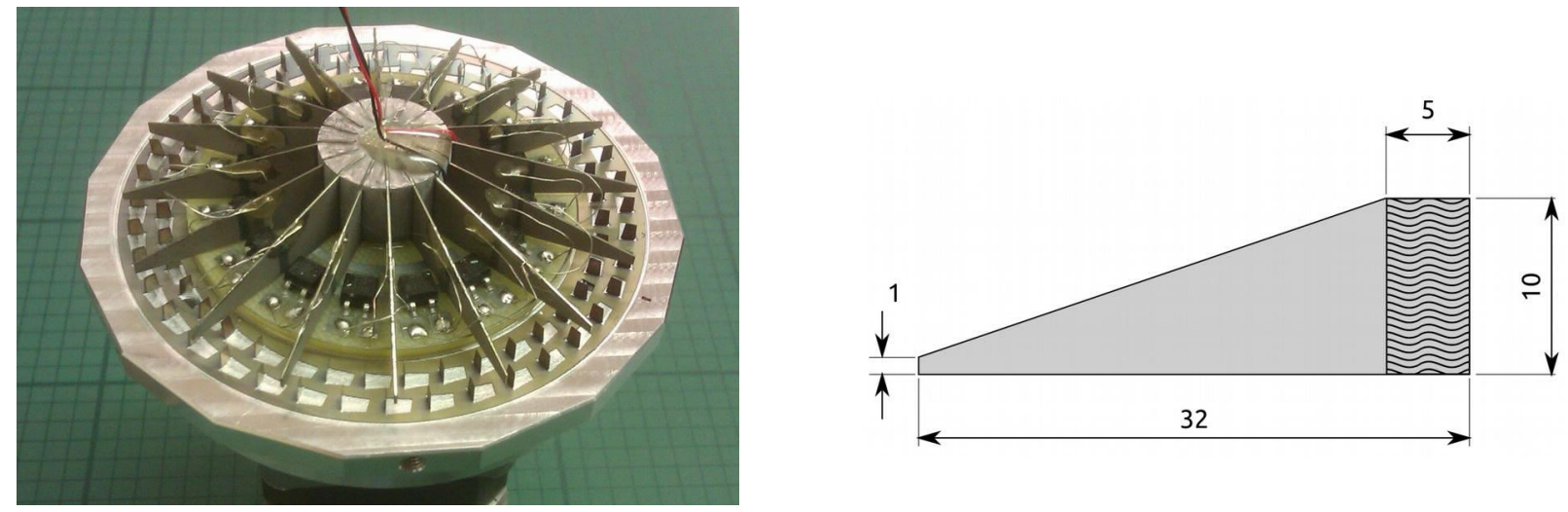

Figure 2: (left) the EH with steel plectra as mounted on the servomotor for testing and (right) the geometry of the bimorphs (the wavy pattern indicates the area fixed into the hub).

with a photoresist. Such coatings can be achieved by laminating, dipping, spinning or rolling processes. The phototool is applied to the resist-coated surface and both exposed to UV light from an appropriate source. As a result, according to the tonality of the phototool and the nature of the photoresist (positive-working or negative working), certain regions of the photoresist will become resistant to or solubilised in a developing solution and thereby either remain on the metal surface or be washed out. Eitherway, selective regions of the metal surface will now be exposed to the action of a suitable etchant (applied by dipping or spraying) and active dissolution will occur as per Figure 1.

As can be seen in Figure 1, the etching of metals is an isotropic process and a degree of "undercut" will be apparent on the finished etched article. The final stage is to strip the photoresist from the metal using an immersion or spraying process. PCM is capable of producing geometries irrespective of the complexity in generally planar substrates.

\section{Manufacture}

Due to the small number of bimorphs required, these were simply obtained by cutting with a diamond saw commercially available rectangular bimorphs (see Figure 2). These are series bimorphs made of a $130 \mu \mathrm{m}$-thick brass layer sandwiched between two PZT-5H, $125 \mu \mathrm{m}$-thick active layers. The root was coated with insulating varnish and fixed into the hub

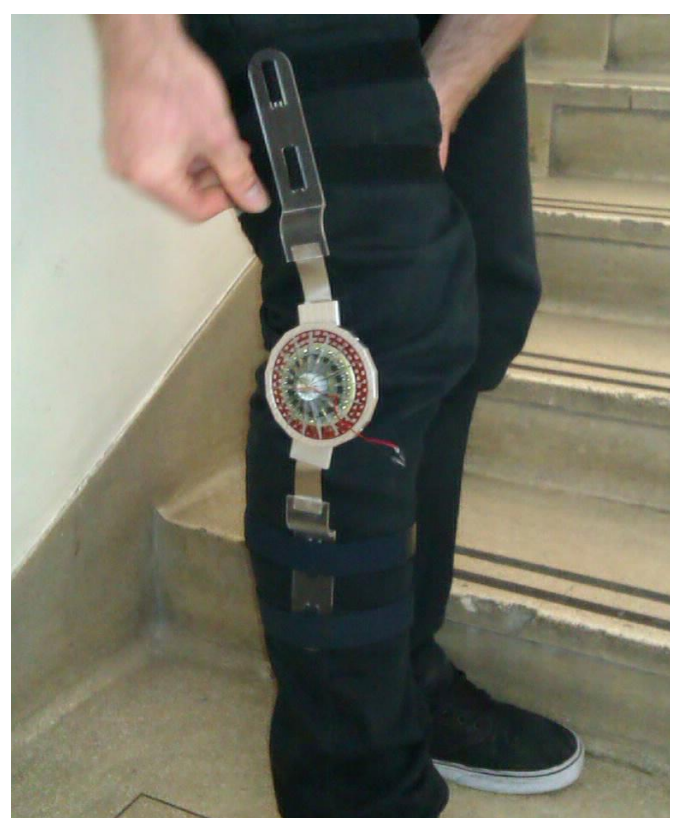

Figure 3: The EH, with kapton plectra, being fitted for testing 

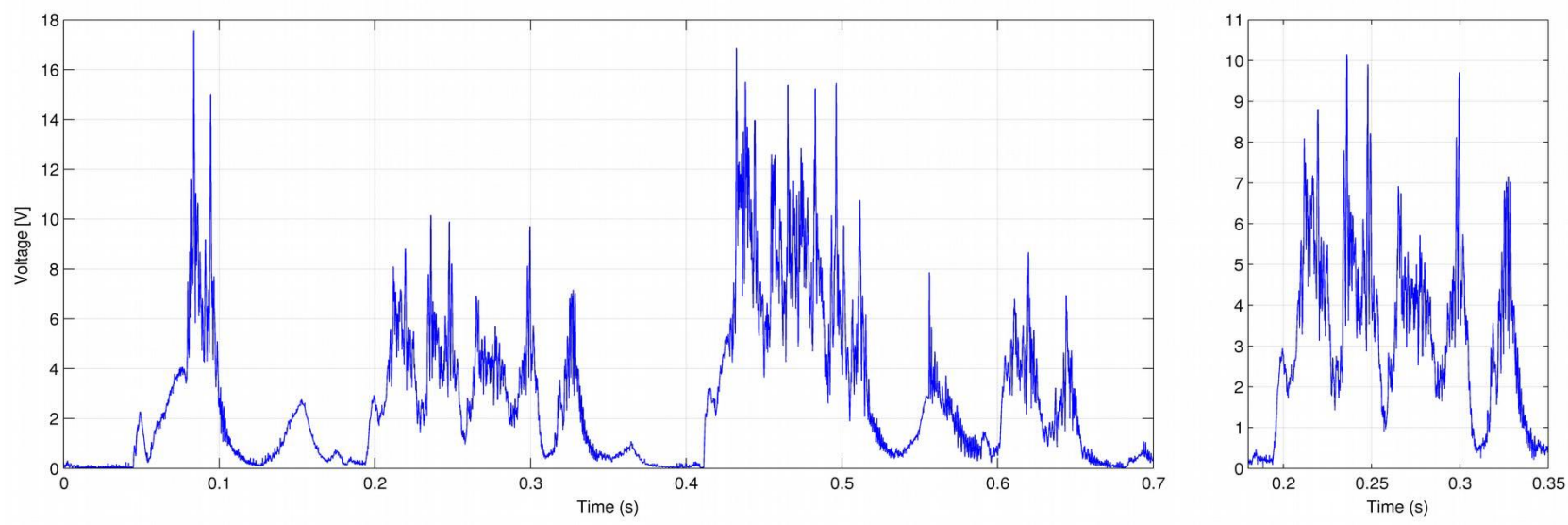

Figure 4: Voltage across load resistor $(21.5 \mathrm{k} \Omega)$ for a run gait with 80 plectra. The detail on the right shows bursts of generation.

with cyanoacrylate adhesive. Wires were connected to both sides of each bimorph with conducting paint and a top layer of polyurethane coating. The other ends were soldered to a custom made printed circuit board (PCB) featuring 16 independent rectifying bridges (Bourns CDNBS04-B08200). The complete device is shown in Figure 2.

For the manufacture of steel plectra, a $100 \mu \mathrm{m}$-thick steel sheet was used. The plectra form two concentric rows of 40 plectra each, shifted so that the 80 plectra are equally separated by $4.5^{\circ}$; the nominal dimensions of the plectra are $3 \mathrm{~mm}$ $\times 2 \mathrm{~mm}$ (height $\times$ width).

AISI 304 stainless steel sheet was immersed in "Micro-90" cleaning solution for several days to remove any contaminant matter from the surface. It was coated using a dry-film laminator $\left(90^{\circ} \mathrm{C}\right.$ and 3 bar pressure) using DuPont MultiMaster MM540 photoresist. UV exposure took place on a Riston UV Exposure unit (5kW for $2.8 \mathrm{~s}$ ). Spray development was then carried out using 1\% (vol) sodium carbonate for 3 mins at ambient temperature. Etching took place in a low pressure ( $<1$ bar) spray etcher for 35 mins in $38^{\circ}$ Bé ferric chloride (aq) at $45^{\circ} \mathrm{C}$.

After machining, the plectra were lifted off and bent at $90^{\circ}$ from the plane of the steel sheet. To remove any possibility of short-circuiting different bimorphs during plucking, the plectra were passivated with a $100 \mathrm{~nm}$ layer of $\mathrm{ZrO}_{2}$.

As an alternative material for the plectra, $125 \mu$ m-thick kapton films were kindly supplied by GTS Flexible Materials
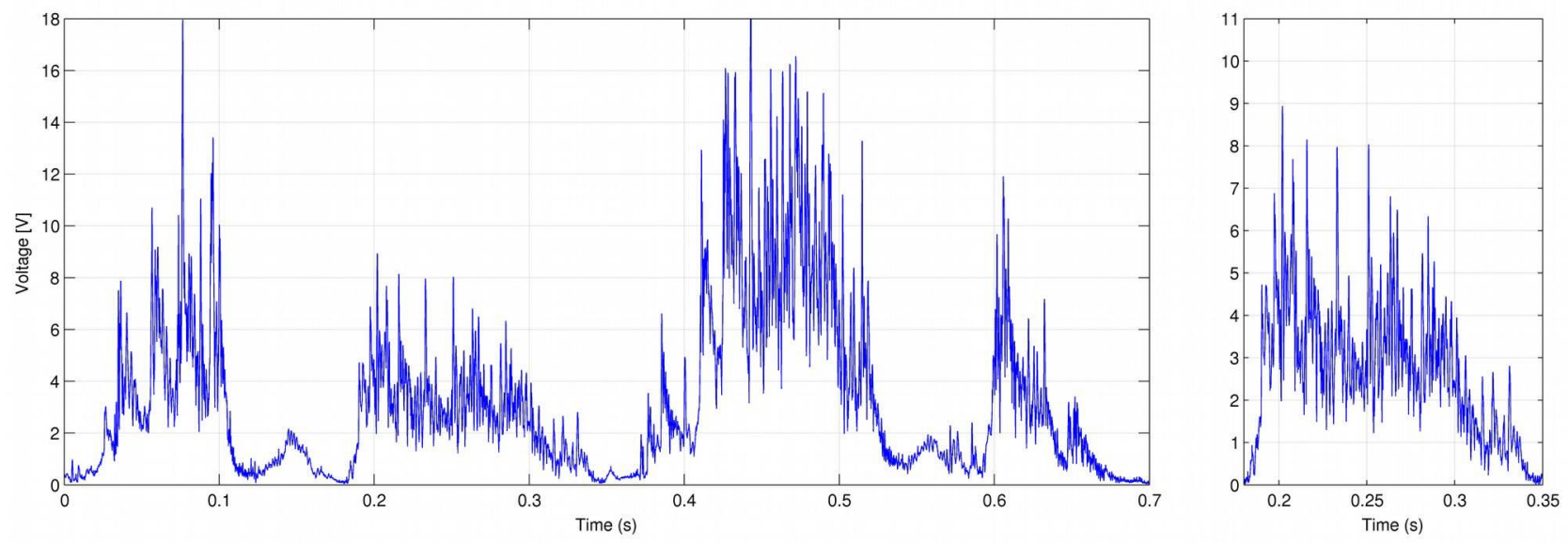

Figure 5: Voltage across load resistor $(21.5 \mathrm{k} \Omega)$ for a run gait with 78 plectra. The detail on the right shows a more continuous generation (as compared to Figure 4) 


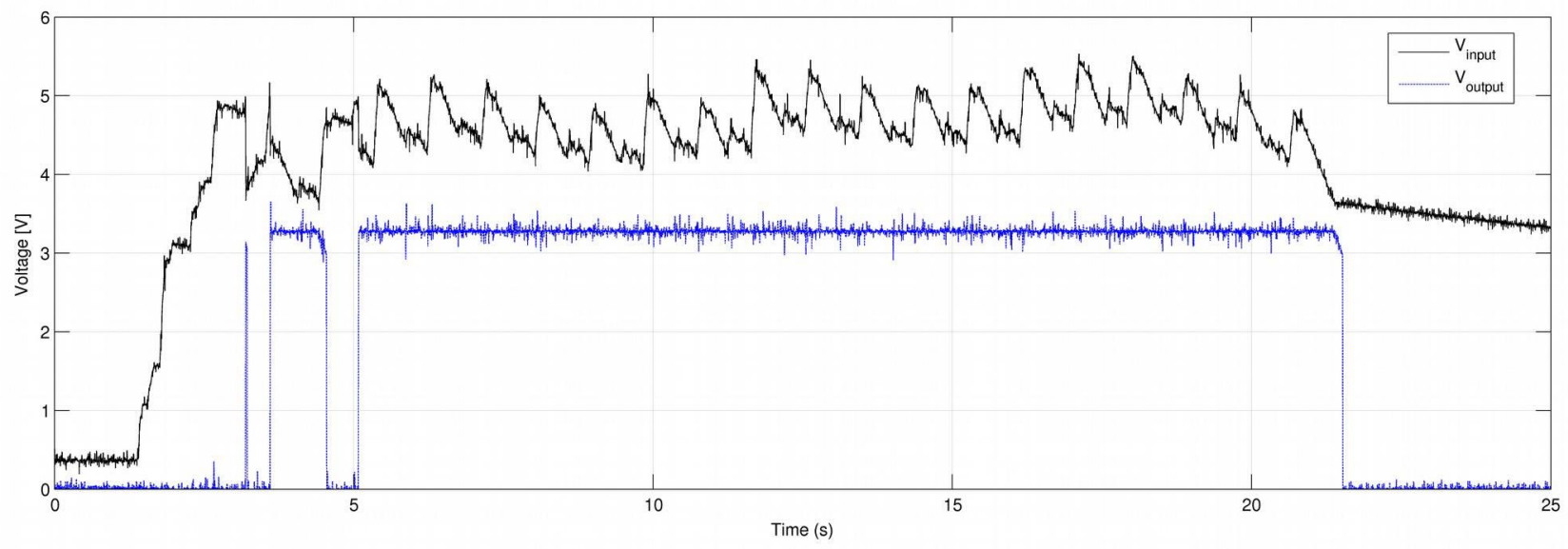

Figure 6: Voltage produced by the harvester (and input into the PMU) and voltage output by the PMU over a $32 \mathrm{k} \Omega$ resistor.

Ltd (Gwent - UK). Initially, the same drawing used for PCM was also used to cut the kapton film with a plotter cutter (4 passes, $90 \mathrm{~mm} / \mathrm{s}$ and $150 \mathrm{~N}$ contact force). However, as the number of plectra in this design is an exact multiple of the number of bimorphs (80 and 16), it was noted that short bursts of generation were separated by periods of inactivity. To avoid simultaneous plucking, a new plectra ring was designed, featuring 2 concentric rows of 39 plectra each (limited space did not permit an increase); as before, the two rows are shifted so that the 78 plectra are uniformly spaced along the circumference.

\section{Experimental methods}

During bench testing, repeatability of the gait was ensured by the use of a basic knee-joint simulator, formed by the combination of an intelligent servomotor commanded by a sequence of positions extracted from gait measurements. In particular, this work focussed on the comparison of energy and power outputs for "walk" and "run" gaits"

The electrical load consisted of a resistor placed at the output of the EH; including the parallel load of the digital acquisition card, the equivalent resistance was $21.5 \mathrm{k} \Omega$ for tests with the kapton plectra and $14.7 \mathrm{k} \Omega$ with the steel plectra. It is to be noted that extensive tests carried out with kapton plectra showed no (statistically) significant difference in energy produced within a load range from 10 to $30 \mathrm{k} \Omega$.

A Power Management Unit (PMU) was developed around the chip LTC3588-1 and configured for a $3.3 \mathrm{~V}$ output. This was used as an alternative load for the EH to test the potential for driving a real load requiring stabilised supply.
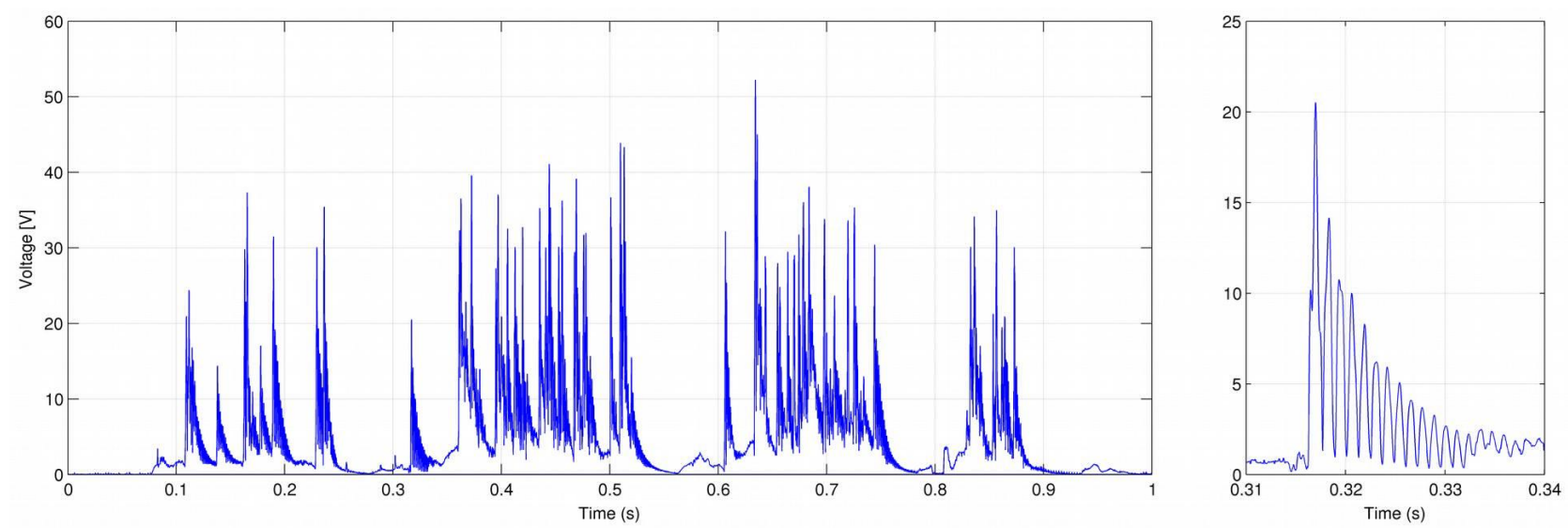

Figure 7: Walking gait with steel plectra (the plot on the right shows a detail) 

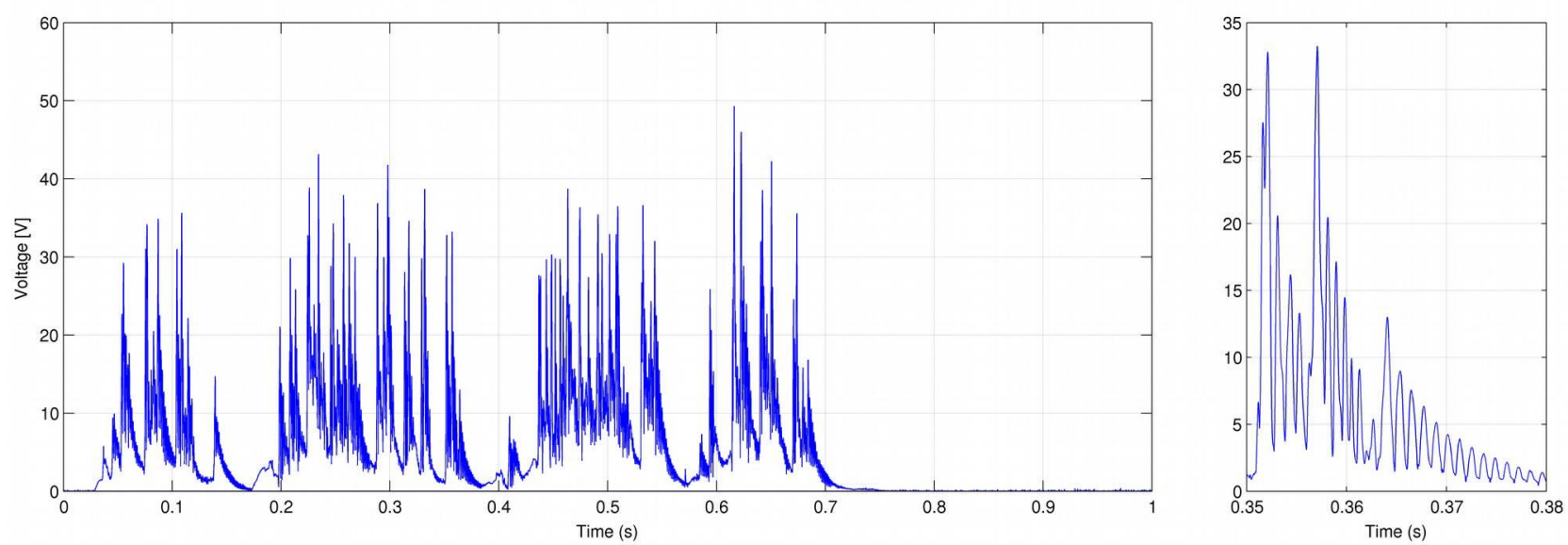

Figure 8: Running gait with steel plectra (the plot on the right shows a detail)

Data acquisition was via a National Instrument NI $9221 \mathrm{~A} / \mathrm{D}$ converter, capable of sampling signals up to $60 \mathrm{~V}$ at 12 bits; acquisition rates of $50 \mathrm{kS} / \mathrm{s}$ were normally used. As this is a benchtop device, a NI USB-6211 connected to a laptop was used for data acquisition during the "in vivo" experiments. In this case, the EH output was dissipated across a voltage divider (of total resistance $21.8 \mathrm{k} \Omega$ ) to produce a measurable voltage within the $\pm 10 \mathrm{~V}$ input limit of the portable DAQ.

Ad hoc braces were designed and manufactured to permit wearing the energy harvester on the lateral side of the knee Figure 3. These were fixed to the thigh and calf by elastic straps, so that a bending of the knee resulted in relative rotation of hub and plate supporting the plectra. The volunteer is a young male of medium build; he walked up and down a short staircase and along a corridor; the energy harvester was fitted with 78 kapton plectra and connected to the voltage divider described above.
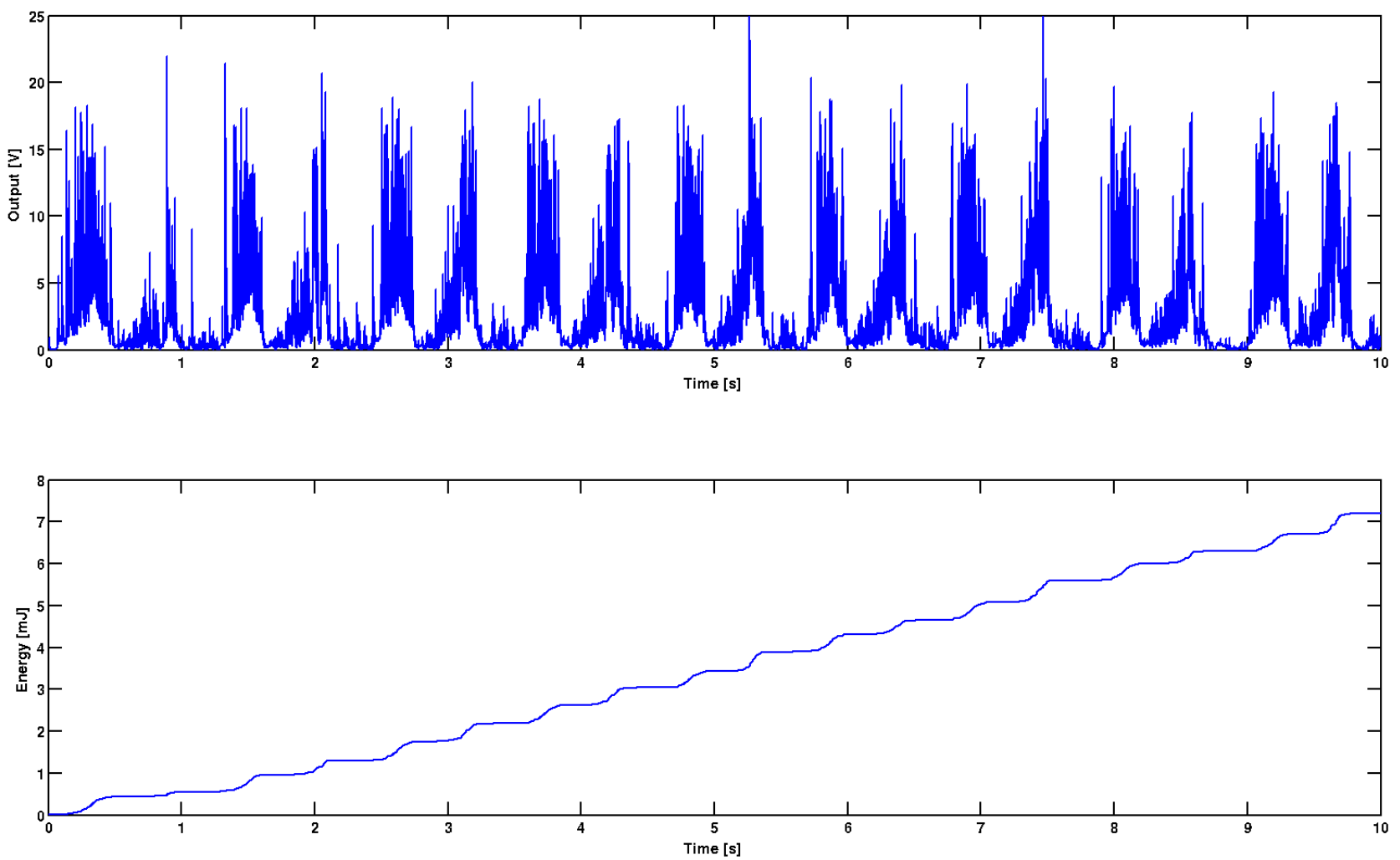

Figure 9: Voltage and energy output from the EH during a 10s walk along a corridor. 


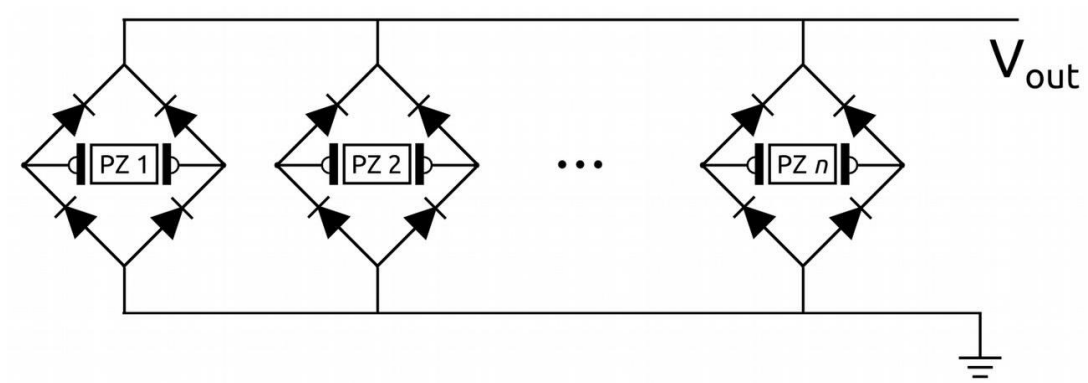

Figure 10: Schematic of the connection of the bimorphs (PZ 1, PZ 2, ..PZ $n$ ) to the output via the rectifying bridges. If the voltage from bimorph $1\left(\mathrm{~V}_{\mathrm{PZ} 1}\right)$ is the highest at a given instant, then $\mathrm{V}_{\text {out }}=\mathrm{V}_{\mathrm{PZ} 1}-2 \times \mathrm{V}_{\mathrm{F}}$ and all other bridges are reversed biased and will not conduct.

\section{EXPERIMENTAL RESULTS}

Figure 4 and Figure 5 reproduce data acquired with running gait on the bench-top simulator on the energy harvester fitted with kapton plectra. The key difference in experimental conditions is that for Figure 480 plectra were fitted, whereas for Figure 5 they were 78. As previously mentioned, 80 being an integer multiple of the number of bimorphs (16), all bimorphs are plucked at the same point in the cycle. This is particularly clear at intermediate speeds, corresponding to the detail shown on the right of each figure. The simultaneous plucking of all 16 bimorphs was audible and a cause of a jerky rotation; a clear improvement was perceived as the plectra were reduced to 78. Interestingly, despite a potentially lower number of plucking actions, the reduction of plectra yielded improved energy generation, as seen in Table 1. The table also shows that changing the overlap between plectra and bimorph by approximately $0.1 \mathrm{~mm}$ around the optimal had no significant effect on the energy produced.

Table 1: Effect of number of kapton plectra and plectrum-bimorph overlap on energy and power generated (given as mean \pm st.dev. of 6 runs)

\begin{tabular}{cccc}
\hline N. of plectra & Overlap [mm] & Energy $[\mathbf{m J}]$ & Power $[\mathbf{m W}]$ \\
\hline 80 & 0.3 & $0.48 \pm 0.02$ & $0.69 \pm 0.03$ \\
\hline 80 & 0.4 & $0.47 \pm 0.02$ & $0.68 \pm 0.03$ \\
\hline 78 & 0.4 & $0.71 \pm 0.06$ & $1.03 \pm 0.08$ \\
\hline
\end{tabular}

For the acquisition of the data graphed in Figure 6, the output of the energy harvester was connected to the input of the PMU introduced above, while the output of the PMU was connected to a $32 \mathrm{k} \Omega$ resistor. The energy harvester was fitted with 78 kapton plectra and a continuous sequence of running gait was commanded to the servomotor. Figure 6 shows that during running, a continuous stable supply exceeding $100 \mu \mathrm{A}$ at $3.3 \mathrm{~V}$ was produced, corresponding to $340 \mu \mathrm{W}$ of electrical energy immediately available for a microcontroller or sensors.

Experiments with the energy harvester fitted with steel plectra produced by photochemical machining focused on the comparison of power produced with walking gait (Figure 7) and running gait (Figure 8). The most obvious difference is that during walking, steps last longer, so that more power can be expected during running than walking. The results in the format mean \pm st.dev. of four tests for each gait are: $4.7 \pm 0.2 \mathrm{~mJ}$ (corresponding to $5.4 \pm 0.3 \mathrm{~mW}$ ) for walking and $5.15 \pm 0.3 \mathrm{~mJ}(7.5 \pm 0.5 \mathrm{~mW})$ for running.

Figure 9 shows $10 \mathrm{~s}$ worth of data collected during a walk along a corridor by the volunteer. The energy harvester was fitted with 78 kapton plectra. The figure shows the voltage across the voltage divider, calculated from the acquired voltage, and the cumulative energy produced. As can be seen, an average power of $720 \mu \mathrm{W}$ was produced in 9 steps. 


\section{DISCUSSION}

In considering data reported in Figure 2, Figure 3 and Table 1, we note that a lower number of plucking actions can yield larger energy generation if they are not concentrated in few events. The reason for this could lie in the rectification process. There are 16 full-bridge rectifiers in the harvester: when one branch of diodes is in conduction, the external circuit will be at this voltage (minus the diode's forward voltage); hence any other bimorph producing a lower voltage will see its bridge reverse biased and will not be able to discharge to the external circuit (see Figure 10). Some of this energy may be transferred later, in a following phase of vibration, but much of it will be lost to damping. When all 16 bimorphs are plucked at approximately the same time, these "collisions" are much more likely than when the plucking actions are non-concurrent.

Table 1 shows that small variations of overlap between kapton plectra and bimorph do not have a measurable effect on the energy generated. This fact has positive repercussions on costs of manufacturing as tolerances can be more relaxed. As regards the amount of energy generated, previous results ${ }^{10}$ entitled to expect higher values. As peak voltages correlate to maximum bimorph deflections, we can infer that in the present studies the bimorphs were plucked less strongly than previously (in previous work ${ }^{10}$ peak voltages of individual bimorphs often exceeded $20 \mathrm{~V}$ ). As the dimensions of the kapton plectra here are nominally identical to those in the cited work and the trapezoidal bimorphs are more compliant, the lower deflections observed in the present work may be due to a less stiff boundary conditions at the root of the plectra (partly due to a weak adhesive bonding between plectra ring and supporting plate) and the fact that contact happens several millimetres closer to the root of the bimorph rather than at the very tip.

From data in Table 1 and Figure 6 it is possible to estimate the efficiency of the PMU. From the table, each running step generates $0.71 \pm 0.06 \mathrm{~mJ}$; close observation of Figure 6 reveals that the step frequency in this test was $1.1 \mathrm{step} / \mathrm{s}$, whereas from gait data the step lasts $0.7 \mathrm{~s}$ (suggesting a frequency of $1.4 \mathrm{step} / \mathrm{s}$ as possible). The difference is due to a limitation of the DAQ system, which introduces a delay during which the data for the next step are uploaded from the PC to the output card. The input power is therefore approximately $780 \mu \mathrm{W}$, yielding an estimate of $44 \%$ for the efficiency of the PMU. It is worth noting that no attempt was made to optimise the discrete components auxiliary to the LT3588-1 chip; furthermore, the power output was set by the selection of voltage and load resistor and no significant capacitive storage was used, so that any extra power available at the input had to be dissipated by the PMU or left in the harvester.

The steel plectra, being of the same areal dimensions and only $25 \mu \mathrm{m}$ thinner, were much stiffer than the kapton plectra, despite the fact that a folding line was also machined at their root to ease their lift off and reduce the stiffness of the constraint. In this case, the overlap plectra-bimorph was significantly more critical and if increased by a few tenths of a millimetres the hub would not be able to rotate. It can be seen from Figure 8 and Figure 7 that peak voltages often approach and occasionally exceed $40 \mathrm{~V}$. Unsurprisingly, this is associated with energy generation 5 times larger than the best achieved with kapton plectra. On the other hand, several plectra broke off after extensive testing, suggesting that an alternative to the folding line should be used. The details on the right show the voltage produced by three bimorphs plucked in rapid succession (Figure 8) and an isolated one (Figure 7).

It is seen from Figure 9 that 9 steps were made in 10 seconds, with an average duration of $1.1 \mathrm{~s} / \mathrm{step}$. This is significantly longer than the walking gait used for bench testing ( $0.88 \mathrm{~s} / \mathrm{step})$ and is partly due to the volunteer walking gingerly because of the lack of habit in wearing a set of braces which proved to be somewhat too loose on the leg and therefore subject to excessive movement. However, the energy of $0.8 \mathrm{~mJ} / \mathrm{step}$ for a walking gait is in good agreement with data from bench tests.

\section{CONCLUSIONS}

The new version of the Pizzicato knee-joint energy harvester presented here has demonstrated that the device can be designed for ease of industrial manufacturing. The power output when fitted with kapton plectra is below expectation; likely causes have been identified and could be easily addressed in a production design. It was found that it is preferable for bimorphs not to be plucked simultaneously, therefore calling for the number of plectra and bimorphs to be relatively prime, if possible. The energy produced by the energy harvester increased dramatically with steel plectra, which afford more vigorous plucking. On the other hand, plectra-bimorph overlap, and therefore hub alignment, are much more critical, requiring more stringent manufacturing tolerances. 
A Power Management Unit (PMU) was developed which permitted to prove that the energy harvester with kapton plectra is capable of delivering a constant supply of $0.1 \mathrm{~mA}$ at $3.3 \mathrm{~V}$ during a sequence of running steps. As the efficiency of the PMU was calculated at $44 \%$, a supply of $1 \mathrm{~mA}$ at $3.3 \mathrm{~V}$ could be achieved during running with steel plectra. Increased performance could also be delivered by optimising the PMU.

\section{ACKNOWLEDGEMENTS}

The authors would like to acknowledge the financial support of CranfieldNano (part of the EPSRC Bridging the Gaps Initiative), which funded part of this work. Newcastle University is also acknowledge for funding the Research Scholarship of Rory Moriarty.

\section{REFERENCES}

[1] Jansen, A. J.., Stevels, A. L. N., “Human power, a sustainable option for electronics,” Electron. Environ. 1999 ISEE -1999 Proc. 1999 IEEE Int. Symp. On, 215-218 (1999).

[2] Mitcheson, P. D., Yeatman, E. M., Rao, G. K., Holmes, A. S.., Green, T. C., "Energy Harvesting From Human and Machine Motion for Wireless Electronic Devices,” Proc. IEEE 96(9), 1457-1486 (2008).

[3] Renaud, M., Elfrink, R., Jambunathan, M., Nooijer, C. de., Wang, Z., Rovers, M., Vullers, R.., Schaijk, R. van., "Optimum power and efficiency of piezoelectric vibration energy harvesters with sinusoidal and random vibrations,” J. Micromechanics Microengineering 22(10), 105030 (2012).

[4] Samson, D., Kluge, M., Becker, T.., Schmid, U., "Wireless sensor node powered by aircraft specific thermoelectric energy harvesting,” Sens. Actuators Phys. 172(1), 240-244 (2011).

[5] Kim, S., Vyas, R., Bito, J., Niotaki, K., Collado, A., Georgiadis, A.., Tentzeris, M. M., “Ambient RF Energy-Harvesting Technologies for Self-Sustainable Standalone Wireless Sensor Platforms,” Proc. Ieee 102(11), 1649-1666 (2014).

[6] Umeda, M., Nakamura, K.., Ueha, S., “Analysis of the Transformation of Mechanical Impact Energy to Electric Energy Using Piezoelectric Vibrator,” Jpn. J. Appl. Phys. 35(Part 1, No. 5B), 3267-3273 (1996).

[7] Kulah, H.., Najafi, K., “An electromagnetic micro power generator for low-frequency environmental vibrations,” Micro Electro Mech. Syst. 2004 17th IEEE Int. Conf. MEMS, 237-240, IEEE (2004).

[8] Renaud, M., Fiorini, P., van Schaijk, R.., van Hoof, C., "Harvesting energy from the motion of human limbs: the design and analysis of an impact-based piezoelectric generator,” Smart Mater. Struct. 18(3), 035001 (2009).

[9] Pillatsch, P., Yeatman, E. M.., Holmes, A. S., “A scalable piezoelectric impulse-excited energy harvester for human body excitation,” Smart Mater. Struct. 21(11), 115018 (2012).

[10] Pozzi, M., Aung, M. S. H., Zhu, M., Jones, R. K.., Goulermas, J. Y., “The pizzicato knee-joint energy harvester: characterization with biomechanical data and the effect of backpack load,” Smart Mater. Struct. 21(7), 075023 (2012).

[11] Goldschmidtboeing, F.., Woias, P., "Characterization of different beam shapes for piezoelectric energy harvesting,” J. Micromechanics Microengineering 18(10), 104013 (2008).

[12] Allen, D. M., “The Principles and Practice of Photochemical Machining and Photoetching”, Adam Hilger, Bristol, England ; Boston (1986).

[13] Diedrich, F. J.., Warren, W. H., Jr., "Why change gaits? Dynamics of the walk-run transition,” J. Exp. Psychol. Hum. Percept. Perform. 21(1), 183-202 (1995). 
2015-05-21

Low-profile and wearable energy

harvester based on plucked

piezoelectric cantilevers

\section{Pozzi, Michele}

Society of Photo-optical Instrumentation Engineers

Pozzi M, Almond HJA, Leighton GJT, Moriarty RJ (2015) Low-profile and wearable energy harvester based on plucked piezoelectric cantilevers, Proceedings of SPIE 9517, Smart

Sensors, Actuators, and MEMS VII; and Cyber Physical Systems, 4-6 May 2015, Barcelona, Spain.

http://dx.doi.org/10.1117/12.2179574

Downloaded from Cranfield Library Services E-Repository 